\title{
Quantum many-body theory of qubit decoherence in a finite-size spin bath
}

\author{
Wen Yang and Ren-Bao Liu* \\ Department of Physics, The Chinese University of Hong Kong, Shatin, N. T., Hong Kong, China
}

\begin{abstract}
Decoherence of a center spin or qubit in a spin bath is essentially determined by the many-body bath evolution. We develop a cluster-correlation expansion (CCE) theory for the spin bath dynamics relevant to the qubit decoherence problem. A cluster correlation term is recursively defined as the evolution of a group of bath spins divided by the cluster correlations of all the subgroups. The so-defined correlation accounts for the authentic (non-factorizable) collective excitations within a given group. The bath propagator is the product of all possible cluster correlation terms. For a finite-time evolution as in the qubit decoherence problem, a convergent result can be obtained by truncating the expansion up to a certain cluster size. The two-spin cluster truncation of the CCE corresponds to the pair-correlation approximation developed previously [Phys. Rev. B 74, 195301 (2006)]. In terms of the standard linked cluster expansion, a cluster correlation term is the infinite summation of all the connected diagrams with all and only the spins in the group flip-flopped, and thus the expansion is exact whenever converges. When the individual contribution of each higher-order correlation term to the decoherence is small (while all the terms combined in product could still contribute substantially), as the usual case for relatively large baths where the decoherence could complete well within the bath spin flip-flop time, the CCE coincides with the cluster expansion [Phys. Rev. B 74, 035322 (2006)]. For small baths, however, the qubit decoherence may not complete within the bath spin flip-flop timescale and thus individual higher-order cluster correlations could grow significant. In such cases, only the CCE converges to the exact coherent dynamics of multi-spin clusters. We check the accuracy of the CCE in an exactly solvable spin-chain model.
\end{abstract}

PACS numbers: 76.20.+q, 03.65.Yz, 76.60.Lz, 76.30.-v

\section{INTRODUCTION}

The decoherence of a center spin in a spin bath ${ }^{1}$ has been of interest in spin resonance spectroscopy for a long history $2.3,4.5$ and is also a paradigmatic model in studying the state collapse in quantum mechanics. Recent revisited interest in this problem is mostly due to the decoherence issue in quantum computing. ${ }^{6.7 .8}$ Most relevant are single electron spins in quantum dots or impurity centers where the dominating decoherence mechanism at low temperatures (such as below a few Kelvins) is the nuclear spins of the host lattice. . $10,11,12,13,14,15,16,17,18,19,20,21,22,23,24,25,26,27,28,29,30,31,32,33,34^{2}$ In a small system such as a quantum dot, the center spin (hereafter referred to as qubit for clarity) and the spin bath, in the time-scale of decoherence, is a relatively isolated subsystem in the whole environment. Thus the qubit decoherence is due to the entanglement with the bath during the coherent evolution of the whole system $\underline{20}^{20}$

In this paper, we are interested in the so-called pure dephasing in which the qubit experiences no longitudinal relaxation but only loses its off-diagonal phase coherence. The pure dephasing is relevant to an electron spin under a moderate or strong magnetic field ( $~ 00.1 \mathrm{~T}$ for a typical GaAs dot), where the electron spin flip due to the hyperfine interaction is largely suppressed by the Zeeman energy mismatch between the electron and the nuclei. In the absence of qubit flip, a qubit-bath system has a Hamiltonian as

$$
H=|+\rangle H^{(+)}\langle+|+|-\rangle H^{(-)}\langle-|,
$$

by which the spin bath is driven by different Hamiltonians $H^{( \pm)}$depending on the qubit states $| \pm\rangle$. When a coherent qubit state $C_{+}|+\rangle+C_{-}|-\rangle$is prepared, the initial state of the qubitbath system is the product state $\left(C_{+}|+\rangle+C_{-}|-\rangle\right) \otimes|\mathcal{J}\rangle$. At time $T$, the bath evolution $|\mathcal{J}\rangle \rightarrow\left|\mathcal{J}^{ \pm}(T)\right\rangle \equiv e^{-i H^{( \pm)} T}|\mathcal{J}\rangle$ predicated on the qubit state $| \pm\rangle$ establishes an entangled state $C_{+}|+\rangle \otimes$ $\left|\mathcal{J}^{+}(T)\right\rangle+C_{-}|-\rangle \otimes\left|\mathcal{J}^{-}(T)\right\rangle$. The qubit coherence is reduced from $\rho_{+-}(0)=C_{+} C_{-}^{*}$ to $\rho_{+-}(T)=C_{+} C_{-}^{*}\left\langle\mathcal{J}^{-}(T) \mid \mathcal{J}^{+}(T)\right\rangle$. The decoherence is characterized by the bath state overlap $\left\langle\mathcal{J}^{-}(T) \mid \mathcal{J}^{+}(T)\right\rangle$. Thus the key is the many-body bath dynamics caused by the interaction within the bath. Without manybody interactions, the bath would not evolve (except for a trivial phase factor that can be eliminated by standard spinecho ${ }^{35}$ ) and the qubit coherence would not decay.

Recently, a variety of quantum many-body theories for nuclear spin bath dynamics have been developed including

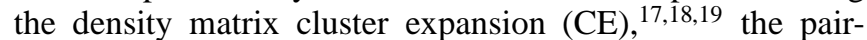
correlation approximation, ${ }^{20,21,22}$ and the linked-cluster expansion (LCE) $\stackrel{23}{=}$ In the pair-correlation approximation, each pair-wise flip-flop of nuclear spins is identified as an elementary excitation mode and is taken as independent of each other. To study the higher oder correlations, the Feynman diagram LCE is developed. The evaluation of higher-order LCE, however, is rather tedious due to the increasing number of diagrams, especially for spins higher than $1 / 2$ (see the Appendix for details). The density matrix CE is developed in the spirit of the standard cluster expansion or virial expansion for interacting gases in grand canonical ensembles $\frac{36,37,38}{3}$ It serves as a simple method (without the need to count or evaluate Feynman diagrams) to include the higher-order spin interaction effects beyond the pair-correlation approximation. The CE calculations show that when the pair-correlation contribution to the decoherence is suppressed by pulse control,, 1 the higher-order correlations are not negligible $\frac{19}{}$ In order to obtain the decoherence exponent from the $\mathrm{CE}$, however, the terms involving overlapping clusters have to be neglected, which make the accuracy problematic even when the expansion converges. This problem limits the $\mathrm{CE}$ to applications in large baths in which the contribution of each individual clus- 
ter to the decoherence is small and the overlapping correction is unimportant.

In this work, we develop a cluster-correlation expansion (CCE) method in which the bath spin evolution are factorized into cluster correlations. Each cluster correlation term is equivalent to the sum of all the LCE series ${ }^{23}$ consisting of a given set of bath spins flip-flopped. In particular, the two-spin cluster correlations include all diagrams with two spins flip-flopped and is equivalent to the pair-correlation approximation. ${ }^{20,21,22}$ The CCE bears the accuracy of the LCE (the results are accurate whenever converge) and the simplicity of the CE (without the need to count or evaluate Feynman diagrams), while free from the large-bath restriction of the CE. The CCE coincides with the CE in the leading order of the short-time expansion, which is applicable in large spin baths where the decoherence completes well within the bath spin flip-flop time. For small baths, however, the qubit decoherence may not complete within the bath spin flip-flop time and individual higher-order cluster correlations could grow significant. In this case only the CCE converges to the exact coherent dynamics of multi-spin clusters. Such coherent dynamics of small clusters of bath spins is of special interest in systems with randomized qubit-bath couplings. An interesting example is nitrogen-vacancy centers in diamonds $39,40,41,42$ which are coupled to randomly located bath spins (carbon-13 and nitrogen nuclear spins) in the proximity.

This paper is organized as follows. In Section II, we derive, for a generic spin bath Hamiltonian, the CCE from a recursive cluster factorization procedure. We show that the CCE is equivalent to an infinite resummation of the LCE series and also compare it to the CE. In section III, we check the accuracy and convergence of the truncated CCE in an exactly solvable model (the one-dimensional spin-1/2 XY model). Section IV draws the conclusions. The Appendix contains the details of the LCE for spin bath dynamics, extended to spins higher than $1 / 2$.

\section{CLUSTER-CORRELATION EXPANSION}

\section{A. Motivation: Pair-correlation approximation and beyond}

As discussed in the Introduction, the Hamiltonian for the pure dephasing problem has the form of Eq. (1). For a given initial bath state $|\mathcal{J}\rangle$ (which could be considered as one sample chosen from a thermal ensemble), the qubit coherence is characterized by

$$
\mathcal{L}(T)=\left\langle\mathcal{J}\left|e^{i H^{(-)} T} e^{-i H^{(+)} T}\right| \mathcal{J}\right\rangle .
$$

For a thermal ensemble of baths, a further ensemble average should be processed. The thermal fluctuation leads to the inhomogeneous broadening, which can be eliminated by spin echo. To focus on the qubit decoherence due to the quantum dynamics of the bath, throughout this paper, we consider the decoherence for a single bath state without the ensemble average. For temperatures much higher than the bath spin flip-flop rates $\left(\sim 10^{-9} \mathrm{~K}\right.$ for nuclear spins in GaAs), the thermal ensemble has no off-diagonal coherence and $|\mathcal{J}\rangle$ can be taken as a (a)

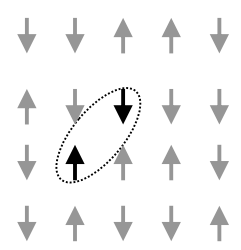

(b)

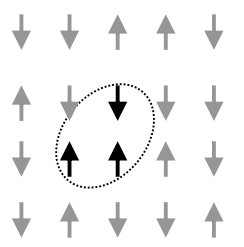

FIG. 1: Visualization of a cluster containing (a) two or (b) three bath spins (black arrows). The spins outside the cluster (gray arrows) are taken as frozen in calculating the cluster contribution.

noninteracting product state $|\mathcal{J}\rangle=\otimes_{n}\left|j_{n}\right\rangle$, where $j_{n}$ denotes the quantum number of the $n$th bath spin quantized along the external magnetic field.

In this subsection we illustrate the central idea of the CCE method (as an extension of the previously developed paircorrelation approximation ${ }^{20,21,22}$ ) using a bath consisting of $N$ spins $\mathbf{J}_{1}, \mathbf{J}_{2}, \cdots$, and $\mathbf{J}_{N}$ with only pairwise secular interactions

$$
\begin{aligned}
H^{( \pm)}= & \pm \frac{\Omega}{2}+\sum_{n}\left(Z_{n} \pm \frac{z_{n}}{2}\right) J_{n}^{z}+\sum_{m \neq n}\left(D_{m, n} \pm \frac{d_{m, n}}{2}\right) J_{m}^{z} J_{n}^{z} \\
& +\sum_{m \neq n}\left(B_{m, n} \pm \frac{b_{m, n}}{2}\right) J_{m}^{+} J_{n}^{-},
\end{aligned}
$$

where $\Omega\left(Z_{n}\right)$ is the qubit (bath spin) splitting energy, $z_{n}$ is the diagonal qubit-bath spin interaction constant (corresponding to the hyperfine interaction strength in an electron-nuclear spin system), $D_{n, m}\left(B_{n, m}\right)$ is the diagonal (off-diagonal) intrinsic bath interaction strength, and $d_{n, m}\left(b_{n, m}\right)$ is the diagonal (off-diagonal) extrinsic bath interaction depending on the qubit states (which could result from the interaction mediated by virtual flips of the qubit spin while real flips are suppressed by the large energy mismatch ${ }^{20}$ ).

In the pair-correlation approximation, the qubit coherence is given by the product of all possible spin pair contributions up to a phase factor, 20

$$
\mathcal{L} \approx \prod_{\{i, j\}} L_{\{i, j\}},
$$

where the contribution due to the flip-flops of a spin pair $\{i, j\}$ [see Fig.11(a)] is

$$
L_{\{i, j\}}=\left\langle\mathcal{J}\left|e^{i h_{\{i, j\}}^{(-)} T} e^{-i h_{\{i, j\}}^{(+)} T}\right| \mathcal{J}\right\rangle,
$$

with the Hamiltonian governing the pair dynamics obtained from the full Hamiltonian $H^{( \pm)}$by excluding the flip-flops of all spins other than $\mathbf{J}_{i}$ and $\mathbf{J}_{j}$

$$
\begin{aligned}
h_{\{i, j\}}^{( \pm)}= & \pm \frac{\Omega}{2}+\sum_{n}\left(Z_{n} \pm \frac{z_{n}}{2}\right) J_{n}^{z}+\sum_{m \neq n}\left(D_{m, n} \pm \frac{d_{m, n}}{2}\right) J_{m}^{z} J_{n}^{z} \\
& +\left(B_{i, j} \pm \frac{b_{i, j}}{2}\right)\left(J_{i}^{+} J_{j}^{-}+J_{i}^{-} J_{j}^{+}\right),
\end{aligned}
$$


which is equivalent to replacing in the full Hamiltonian the spins outside the pair with their mean-field averages,

$$
h_{\{i, j\}}^{( \pm)} \equiv H^{( \pm)}\left(\mathbf{J}_{i}, \mathbf{J}_{j},\left\{\left\langle\mathcal{J}\left|\mathbf{J}_{n \neq i, j}\right| \mathcal{J}\right\rangle\right\}\right) .
$$

The pair-correlation approximation is valid for situations where pair correlations dominate. When the collective flipflops of more spins become important, we need to consider the higher-order correlation correction $\mathcal{L}_{\text {corr }}$ defined by

$$
\mathcal{L}=\left(\prod_{\{i, j\}} L_{\{i, j\}}\right) \mathcal{L}_{\text {corr }} .
$$

To illustrate how the high-order correction could be evaluated. let us consider a spin bath of only three spins $\{1,2,3\}$. Obviously, the result is

$$
\mathcal{L}_{\text {corr }}=\frac{\mathcal{L}}{L_{\{1,2\}} L_{\{2,3\}} L_{\{3,1\}}} .
$$

This result motivates a definition of non-factorizable threespin correlations due to collective flip-flops. For a three-spin cluster $\{i, j, k\}$ in a bath [see Fig.11 b)], when all the spins outside the cluster are frozen, the qubit coherence is

$$
L_{\{i, j, k\}}=\left\langle\mathcal{J}\left|e^{i h_{(i, j, k\}}^{(-)} T} e^{-i h_{i, j, k\}}^{(+)} T}\right| \mathcal{J}\right\rangle,
$$

with the cluster Hamiltonian

$$
\begin{aligned}
h_{\{i, j, k\}}^{( \pm)} \equiv & \pm \frac{\Omega}{2}+\sum_{n}\left(Z_{n} \pm \frac{z_{n}}{2}\right) J_{n}^{z}+\sum_{m \neq n}\left(D_{m, n} \pm \frac{d_{m, n}}{2}\right) J_{m}^{z} J_{n}^{z} \\
& +\sum_{m, n \in\{i, j, k\}}\left(B_{m, n} \pm \frac{b_{m, n}}{2}\right) J_{m}^{+} J_{n}^{-} \\
= & H^{( \pm)}\left(\mathbf{J}_{i}, \mathbf{J}_{j}, \mathbf{J}_{k},\left\{\left\langle\mathcal{J}\left|\mathbf{J}_{n \notin\{i, j, k\}}\right| \mathcal{J}\right\rangle\right\}\right),
\end{aligned}
$$

obtained from the full Hamiltonian $H^{( \pm)}$by replacing the spins outside the cluster with their mean-field averages. The authentic (or non-factorizable) three-spin correlation is singled out by excluding all the pair-correlations

$$
\tilde{L}_{\{i, j, k\}}=\frac{L_{\{i, j, k\}}}{L_{\{i, j\}} L_{\{i, k\}} L_{\{j, k\}}} .
$$

If all such three-spin correlations are picked up, the qubit coherence is given by (up to a global phase factor)

$$
\mathcal{L} \approx \prod_{\{i, j\}} L_{\{i, j\}} \cdot \prod_{\{i, j, k\}} \tilde{L}_{\{i, j, k\}} .
$$

Thus a systematic cluster correlation expansion is motivated.

\section{B. General formalism of cluster-correlation expansion}

We consider a generic bath Hamiltonian for the pure dephasing problem

$$
H^{( \pm)}=H^{( \pm)}\left(\mathbf{J}_{1}, \mathbf{J}_{2}, \cdots, \mathbf{J}_{N}\right),
$$

(a)

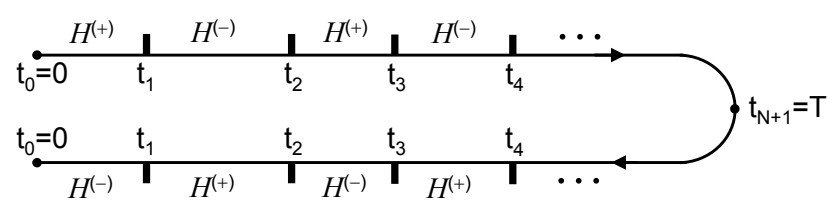

(b)

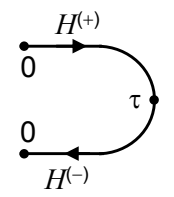

(c)

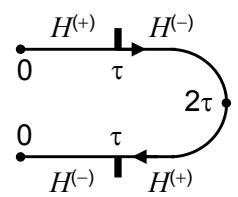

(d)

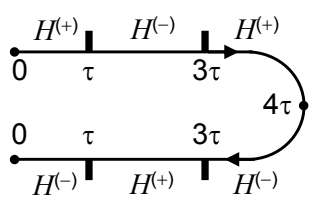

FIG. 2: (a) Visualization of an arbitrary sequence of controlling $\pi$ pulses at $t_{1}, t_{2}, \cdots$, (indicated by vertical lines) and the corresponding contour-time-dependent Hamiltonian for electron spin decoherence. (b), (c), and (d) exemplify the cases of free-induction decay, Hahn echo, and Carr-Purcell echo, respectively.

which need not contain only pairwise interactions, or conserve the spin angular momentum along any direction. For instance, multi-spin interaction terms like $J_{i}^{z} J_{j}^{+} J_{k}^{-}$and nonsecular terms like $J_{i}^{+} J_{j}^{z}$ could be present.

For qubit decoherence under the control of an arbitrary sequence of $\pi$-pulses applied at $t_{1}, t_{2}, \cdots$, as shown schematically in Fig. 2(a), the bath evolution predicated on the qubit state is given by

$$
\left|\mathcal{J}^{ \pm}(T)\right\rangle=U^{( \pm)}|\mathcal{J}\rangle
$$

where

$$
U^{( \pm)} \equiv \cdots e^{-i H^{( \pm)}\left(t_{3}-t_{2}\right)} e^{-i H^{(\mp)}\left(t_{2}-t_{1}\right)} e^{-i H^{( \pm)} t_{1}} .
$$

The qubit coherence at the end of the evolution is

$$
\mathcal{L}=\left\langle\mathcal{J}\left|\left(U^{(-)}\right)^{\dagger} U^{(+)}\right| \mathcal{J}\right\rangle .
$$

It can be written in the contour time-ordered form as $\underline{23}$

$$
\mathcal{L}=\left\langle\mathcal{J}\left|\mathscr{T}_{\mathrm{c}} e^{-i \int_{\mathrm{c}} H(t) d t}\right| \mathcal{J}\right\rangle,
$$

where $\mathscr{T}_{\mathrm{c}}$ is the time-ordering operator along the contour $C: 0 \rightarrow T \rightarrow 0$. As illustrated in Fig. 2(a), the contour time-dependent Hamiltonian $H(t)$ alternatively switches between $H^{(+)}$and $H^{(-)}$each time the qubit state is flipped by a $\pi$-pulse or when the time direction is reverted at $T$. The examples for free-induction decay, single-pulse Hahn echo, and Carr-Purcell echo are illustrated in Figs. 2 (b), (c), and (d), respectively.

Following the idea illustrated in the previous subsection, the cluster correlations are recursively defined as follows.

1. The empty-cluster correlation

$$
\tilde{L}_{\varnothing} \equiv L_{\varnothing} \equiv e^{-i \int_{\complement}\langle\mathcal{T}|H(t)| \mathcal{J}\rangle d t}
$$


is a pure phase factor obtained from Eq. (11) by replacing the bath Hamiltonian $H(t)$ with its mean-field average $\langle\mathcal{J}|H(t)| \mathcal{J}\rangle$.

2. The single-spin correlation

$$
\tilde{L}_{\{i\}} \equiv L_{\{i\}} / \tilde{L}_{\varnothing}
$$

where

$$
L_{\{i\}} \equiv\left\langle\mathcal{J}\left|\mathscr{T}_{\mathrm{c}} e^{-i \int_{\mathrm{c}} h_{\{i\}}(t) d t}\right| \mathcal{J}\right\rangle
$$

is obtained from Eq. (11) by replacing the bath Hamiltonian $H\left(\left\{\mathbf{J}_{n}\right\}, t\right)$ with

$$
h_{\{i\}}(t) \equiv H\left(\mathbf{J}_{i},\left\{\left\langle\mathcal{J}\left|\mathbf{J}_{n \neq i}\right| \mathcal{J}\right\rangle\right\}, t\right) .
$$

in which all the spin operators except $\mathbf{J}_{i}$ are mean-field averaged.

3. The two-spin (pair) correlation

$$
\tilde{L}_{\{i, j\}} \equiv L_{\{i, j\}} /\left(\tilde{L}_{\varnothing} \tilde{L}_{\{i\}} \tilde{L}_{\{j\}}\right)
$$

where

$$
L_{\{i, j\}} \equiv\left\langle\mathcal{J}\left|\mathscr{T}_{\mathrm{c}} e^{-i \int_{\mathrm{c}} h_{\{i, j\}}(t) d t}\right| \mathcal{J}\right\rangle
$$

is obtained from Eq. 111 by replacing the bath Hamiltonian $H\left(\left\{\mathbf{J}_{n}\right\}, t\right)$ with

$$
h_{\{i, j\}}(t) \equiv H\left(\mathbf{J}_{i}, \mathbf{J}_{j},\left\{\left\langle\mathcal{J}\left|\mathbf{J}_{n \notin\{i, j\}}\right| \mathcal{J}\right\rangle\right\}, t\right),
$$

in which all the spin operators except $\mathbf{J}_{i}$ and $\mathbf{J}_{j}$ are mean-field averaged.

4. So on and so forth, the cluster correlation for an arbitrary set of bath spins $C$ is defined as

$$
\tilde{L}_{C} \equiv \frac{L_{C}}{\prod_{C^{\prime} \subset C} \tilde{L}_{C^{\prime}}},
$$

where

$$
L_{C} \equiv\left\langle\mathcal{J}\left|\mathscr{T}_{\mathrm{c}} e^{-i \int_{\mathrm{c}} h_{C}(t) d t}\right| \mathcal{J}\right\rangle
$$

is obtained from Eq. 111 by replacing the bath Hamiltonian $H\left(\left\{\mathbf{J}_{n}\right\}, t\right)$ with

$$
h_{C}(t) \equiv H\left(\left\{\mathbf{J}_{n \in C}\right\},\left\{\left\langle\mathcal{J}\left|\mathbf{J}_{n \notin C}\right| \mathcal{J}\right\rangle\right\}, t\right),
$$

in which all the spin operators outside the cluster are mean-field averaged or their flip-flops are frozen.

Thus, by definition, the qubit coherence is factorized into all possible cluster correlations as

$$
\mathcal{L}=\prod_{C \subseteq\{1,2, \cdots, N\}} \tilde{L}_{C}
$$

Calculating the CCE to the maximum order $\tilde{L}_{\{1,2, \cdots, N\}}$ amounts to solving the exact bath propagator, which is in general not possible. In the decoherence problem, we consider a finitetime evolution and it often suffices to truncate the expansion by keeping cluster correlations up to a certain size $M$, as the $M$ th-order truncation of the CCE ( $M$-CCE for short),

$$
\mathcal{L}^{(M)}=\prod_{|\mathcal{C}| \leq M} \tilde{L}_{C},
$$

where $|C|$ is the number of spins contained in the cluster $C$. As an example, for the pairwise Hamiltonian in Eq. (2), the lowest nontrivial order of truncation is

$$
\mathcal{L}^{(2)}=\tilde{L}_{\varnothing} \prod_{\{i, j\}} \tilde{L}_{\{i, j\}}=\tilde{L}_{\varnothing} \prod_{\{i, j\}}\left(L_{\{i, j\}} / \tilde{L}_{\varnothing}\right),
$$

which is the pair-correlation approximation.

\section{Relation to linked-cluster expansion}

Saikin et al. have recently developed an LCE method for the qubit decoherence in a spin-1/2 bath. ${ }^{23}$ The detailed descriptions of the LCE for a generic spin bath are given in the Appendix. In general, the bath evolution can be factorized using Feynman diagrams so that

$$
\mathcal{L}=\exp (\pi)
$$

where

$$
\pi=\left\langle\mathcal{J}\left|\mathscr{T}_{\mathrm{c}} e^{-i \int_{\mathrm{c}} H(t) d t}\right| \mathcal{J}\right\rangle_{\text {connected }},
$$

is the sum of all connected Feynman diagrams obtained by using Wick's theorem on the series expansion of Eq. (11). Some of the connected diagrams up to the 4th-order have been evaluated in Ref. [23] for free-induction decay and single-pulse Hahn echo with a spin-1/2 bath Hamiltonian in the form of Eq. (2). The complexity for diagram counting and evaluation increases dramatically when considering higher-order diagrams (see Fig. 11 in Appendix A 2) or higher spins [see Fig. 13 (b) in Appendix A3 for the second-order diagrams for a spin-1 bath].

We notice that each diagram can be expanded as the sum of diagrams involving the flip-flops of different clusters of spins. As an example shown in Fig. 3 a), a third-order diagram involving the flip-flop of a spin pair contains diagrams for spin clusters $(1,2),(1,3), \cdots$, where the numbers stand for the indices of the spins flip-flopped (i.e., the $J^{z}$ quantum number changed). Thus all the connected diagrams can be classified according to the spin clusters instead of the interaction orders. For an arbitrary cluster $C$, we define $\tilde{\pi}(C)$ as the sum of all connected diagrams in which all and only the spins in cluster $C$ have been flip-flopped. For instance, some of the diagrams constituting $\tilde{\pi}(\varnothing)$ and $\tilde{\pi}(i, j)$ for a spin-1/2 Hamiltonian in Eq. (2) are shown in Figs. 3(b) and (c), respectively. With these $\{\tilde{\pi}\}$ functions, the LCE is expressed as

$$
\pi=\sum_{C \subseteq\{1,2, \cdots, N\}} \tilde{\pi}(C) .
$$


(a)

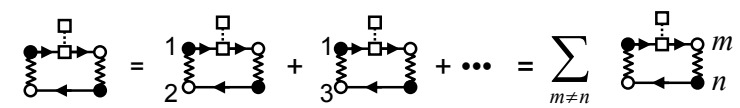

(b) $\tilde{\pi}(\varnothing)=\dot{a}+\stackrel{\square}{\square}$
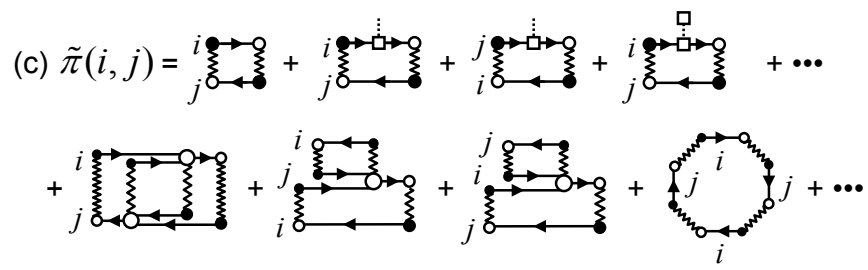

FIG. 3: (a) Expansion of a third-order connected diagram into diagrams involving the flip-flops of different clusters of spins. (b) and (c) show the diagrams contained in $\tilde{\pi}(\varnothing)$ and $\tilde{\pi}(i, j)$, respectively. In the diagrams, a solid arrow denotes the propagation of a spin. A wavy (dotted) line connecting two solid arrows/spins denotes a pairwise off-diagonal (diagonal) interaction, and an open-ended line represents the interaction with the qubit spin or an external field. Each filled circle/empty circle/empty square on a solid arrow denotes a $J^{+} / J^{-} / J^{z}$ operator, which raises/lowers/keeps invariant the $J^{z}$ quantum number of that spin.

In particular, the infinite summation of all the connected diagrams for a certain cluster $C$ and all its subsets

$$
\pi(C) \equiv \sum_{C^{\prime} \subseteq C} \tilde{\pi}\left(C^{\prime}\right)
$$

can be obtained from the series expansion Eq. (19) by dropping all the terms involving the flip-flop of spins outside the cluster $C$, or, equivalently, by reducing the bath Hamiltonian $H(t)$ to the cluster Hamiltonian $h_{C}(t)$ in which the spins outside the cluster are mean-field averaged. Thus we have

$$
e^{\pi(\mathcal{C})}=\prod_{\mathcal{C}^{\prime} \subseteq \mathcal{C}} e^{\tilde{\pi}\left(C^{\prime}\right)}=\left\langle\mathcal{J}\left|\mathscr{T}_{\mathrm{c}} e^{-i \int_{\mathrm{c}} h_{\mathcal{C}}(t) d t}\right| \mathcal{J}\right\rangle=L_{C} .
$$

Comparing this to Eqs. (12)-(14), we immediately have

$$
\tilde{L}_{C}=e^{\tilde{\pi}(\mathcal{C})},
$$

i.e., a cluster correlation term corresponds to the infinite partial summation of all the connected diagrams in which all and only the spins in the cluster have been flip-flopped.

From the LCE expression of the CCE in Eq. (23), it is obvious that the short-time profile of the decoherence due to clusters of a certain size is determined by the lowest-order diagram. In particular, for free-induction decay with the secular pair-interaction Hamiltonian in Eq. (2), the leading order contribution from a cluster of size $M$ is

$$
\tilde{\pi}(C) \sim B^{M} T^{M},
$$

where $B$ is the typical magnitude of the pair flip-flop interaction strength $B_{m, n} \pm b_{m, n}$. If each spin interacts, on average, with $q$ spins, then the number of size- $M$ clusters is $\sim N q^{M-1}$, with $N$ the total number of bath spins. The sum of all the leading order $M$-spin connected diagrams is $\sim q^{-1} N \cdot(q B T)^{M}$.
Thus for $q B T \ll 1$, i.e., for a time $T$ much shorter than the bath spin flip-flop timescale $1 / B$, a truncated CCE converges. The short time condition $T \ll B^{-1}$ is usually satisfied for electron spin decoherence caused by nuclear spins in typical quantum dots. The convergence of the truncated CCE, however, could go well beyond the short time restriction. One such scenario is small spin baths with disorder in qubit-bath couplings or in spin splitting energies, in which multi-spin correlation could develop at a time well beyond the short-time limit but the size of the contributing clusters could remain bounded due to the localization effect in a disordered system, as will be verified later in this paper by numerical simulations.

\section{Relation to cluster expansion}

Witzel et al. recently developed a density matrix CE approach to solving the nuclear spin dynamics in the electron spin decoherence problem, ${ }^{17,19}$ in the spirit of the cluster or virial expansion for interacting gases in grand canonical ensembles ${ }^{36,37,38}$ Below we reproduce the basic procedure of the CE and compare it to the CCE. Instead of the ensemble CE in Ref. [17], we consider a single sample state of the bath for a direct comparison. Defining the qubit decoherence due to a cluster $C$ of bath spins as

$$
W(C) \equiv\left|\left\langle\mathcal{J}\left|\mathscr{T}_{\mathrm{c}} e^{-i \int_{\mathrm{c}} h_{\mathcal{C}}(t) d t}\right| \mathcal{J}\right\rangle\right|=\left|L_{C}\right|,
$$

a hierarchy of cluster terms $\{\tilde{W}(C)\}$ are recursively defined as

$$
\begin{aligned}
W(i)= & \tilde{W}(i), \\
W(i, j)= & \tilde{W}(i, j)+\tilde{W}(i) \tilde{W}(j), \\
W(i, j, k)= & \tilde{W}(i, j, k)+\tilde{W}(i) \tilde{W}(j) \tilde{W}(k)+\tilde{W}(i, j) \tilde{W}(k) \\
& +\tilde{W}(i, k) \tilde{W}(j)+\tilde{W}(i) \tilde{W}(j, k), \\
& \ldots \\
W(C)= & \tilde{W}(C)+\sum_{\left\{C_{j}\right\}} \prod_{\mathcal{C}_{j}} \tilde{W}\left(C_{j}\right),
\end{aligned}
$$

where in the last line the sum runs over all possible partitions of the cluster $C$ into nonoverlapping nonempty subsets $C_{1}, C_{2}, \cdots$. The $M$ th-order truncated CE ( $M$-CE for short) is

$$
W^{(M)}=\sum_{\left.\left\{C_{j}\right\}, \mid \mathcal{C}_{j}\right\} \leq M} \prod_{\mathcal{C}_{j}} \tilde{W}\left(C_{j}\right)
$$

with the sum running over all possible partitions of the bath into nonoverlapping nonempty clusters $C_{1}, C_{2}, \cdots$ of size up to $M$.

In the cluster expansion for interacting gases in grand canonical ensembles with translational symmetry, the contribution from different clusters can be factorized ${ }^{36,37,38}$ and the evaluation of a truncated $\mathrm{CE}$ amounts to the calculation of a finite number of finite-size cluster contributions, similar to the CCE in this paper. For a finite-size spin bath or for a bath without translational symmetry, however, such factorization of different clusters does not exist, which makes it essentially 
impossible to calculate the sum in Eq. 27) even for a small$M$-CE. For example, for a bath of $N$ spins, the number of all terms containing only pair clusters is $O(N ! !)$ and all such terms have to be individually calculated and summed in the 2-CE, which is practically impossible.

A remedy is possible when all the cluster terms $\tilde{W}(C)$ are individually small (but the sum could still be substantial). Under such a condition, the CE can be approximated by a factorized form by adding some overlapping terms which are higherorder small. ${ }^{17}$ For example, with the secular pair-interaction Hamiltonian in Eq. (2), for which $W(i)=\tilde{W}(i)=1$, the $M$-CE is

$$
W^{(M)} \approx \prod_{1<|C| \leq M}[1+\tilde{W}(C)],
$$

under the small-term condition

$$
|\tilde{W}(C)| \ll 1, \text { for }|C|>1 .
$$

Comparing the factorization approximation in Eq. (28) to the exact $M$-CE in Eq. (27), the error added is

$$
\delta W^{(M)}=\sum_{i<j<k} \tilde{W}(i, j) \tilde{W}(j, k)+\sum_{i<j<k<l} \tilde{W}(i, j, k) \tilde{W}(k, l)+\cdots,
$$

containing products of any set of cluster terms sharing at least one spin, i.e., the overlapping terms. Such overlapping terms are higher-order small if each individual cluster term (for $|C|>1$ ) is small. Furthermore, under the small-term condition, $1+\tilde{W}(C) \approx \exp [\tilde{W}(C)]$, and the CE for an arbitrary cluster becomes

$$
W(C) \approx \prod_{C^{\prime} \subseteq C} e^{\tilde{W}\left(C^{\prime}\right)}
$$

which takes the same form as the CCE.

Thus the CCE coincides with the CE under the small-term condition Eq. (29), which is justified for large spin baths where the number of contributing clusters is large and hence the contribution from each individual cluster remains small within the timescale of decoherence. The problem with the neglected overlapping terms is relevant for small spin baths where the coherent dynamics of a small number of multi-spin clusters dominating the decoherence may persist well beyond the bath spin flip-flop time and the small-term condition is no longer satisfied. In this case the $\mathrm{CE}$ will not converge to the exact multi-spin cluster dynamics, as will be seen in the numerical check in the next section.

\section{NUMERICAL CHECK}

Here we consider an exactly solvable spin bath model (the one-dimensional spin-1/2 XY model) and compare the exact solution to the results obtained with the CCE and the CE. The $N$-spin bath Hamiltonian conditioned on the qubit state $| \pm\rangle$ (with spin splitting constants dropped) is

$$
H^{( \pm)}= \pm \sum_{n=1}^{N} \frac{z_{n}}{2} J_{n}^{z}+\sum_{n=1}^{N-1}\left(B_{n} \pm \frac{b_{n}}{2}\right)\left(J_{n+1}^{+} J_{n}^{-}+J_{n}^{+} J_{n+1}^{-}\right)
$$
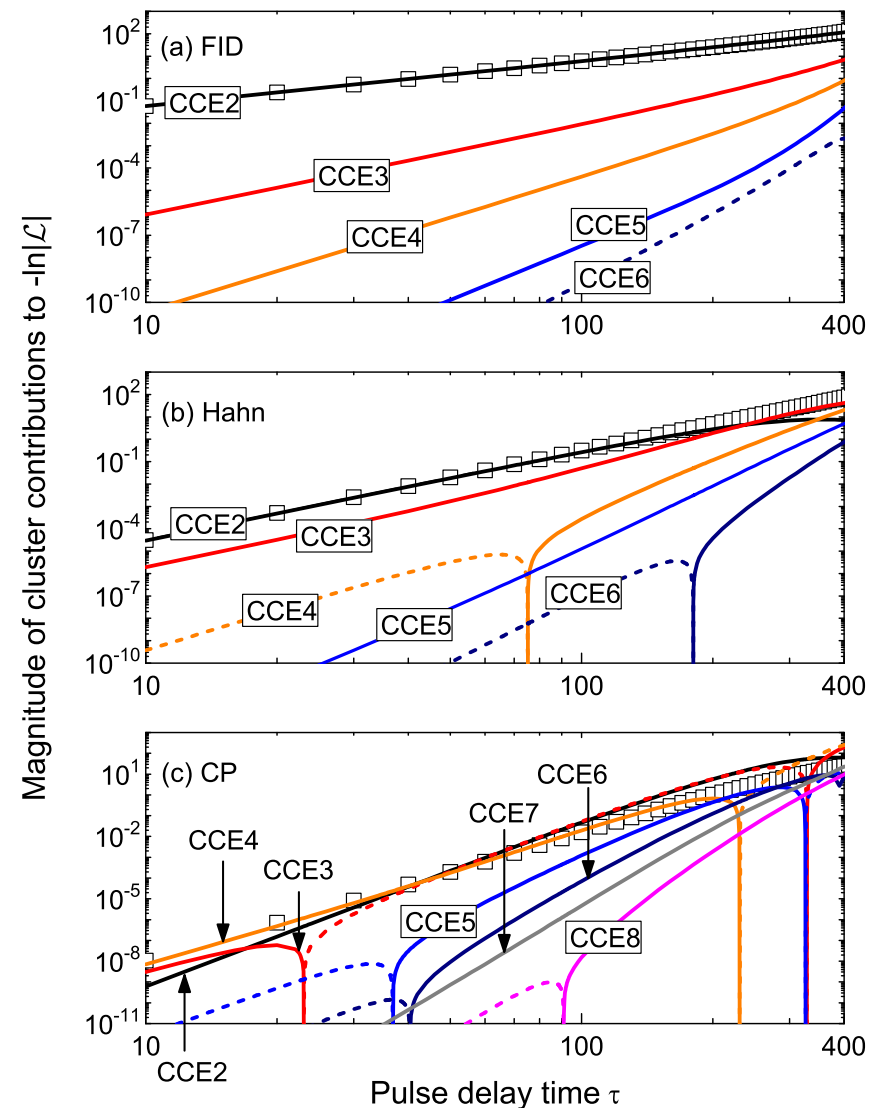

FIG. 4: (Color online) The magnitude of size- $m$ cluster contributions (denoted as CCE $m$ ) to the exponential decoherence factor $-\ln |\mathcal{L}|$ in (a) free-induction decay (FID), (b) Hahn echo, and (c) Carr-Purcell echo (CP) for a long "sinusoidal" spin chain with $N=1000$ spins. A solid (dashed) line indicates that the cluster contribution to $-\ln |\mathcal{L}|$ is positive (negative). The exact $-\ln |\mathcal{L}|$ is also shown (empty squares) for comparison.

where $z_{n}$ denotes the qubit-bath spin interaction coefficient, $B_{n}$ is the intrinsic bath interaction strength, and $b_{n}$ is the interaction dependent on the qubit state. The initial bath state $|\mathcal{J}\rangle$ is taken as a product state of all bath spins, in which the orientation of each bath spin is randomly chosen as up or down. The qubit-bath interaction coefficients $\left\{z_{n}\right\}$ are either taken from a sinusoidal distribution $z_{n}=z_{\max } \sin (n \pi / N)$ (referred to as a "sinusoidal" spin chain) or randomly chosen from $\left[0, z_{\max }\right]$ (referred to as a "random" spin chain). Hereafter $z_{\max }$ is taken as the unit of energy. The spin-flip interaction strengths $\left\{B_{n}\right\}$ and $\left\{b_{n}\right\}$ are randomly chosen from $\left[10^{-3}, 2 \times 10^{-3}\right]$, corresponding to typical bath spin flip-flop time $\tau_{\text {sf }} \sim 10^{3}$. The exact solution is obtained by the Jordan-Wigner transformation, which transforms the interacting spin-1/2 chain to a noninteracting fermion system. 43,44

\section{A. Large spin bath}

As the first example, we consider a sinusoisal chain of $N=1000$ spins. In this case, the energy cost $\left|z_{n}-z_{n+1}\right|$ for a 


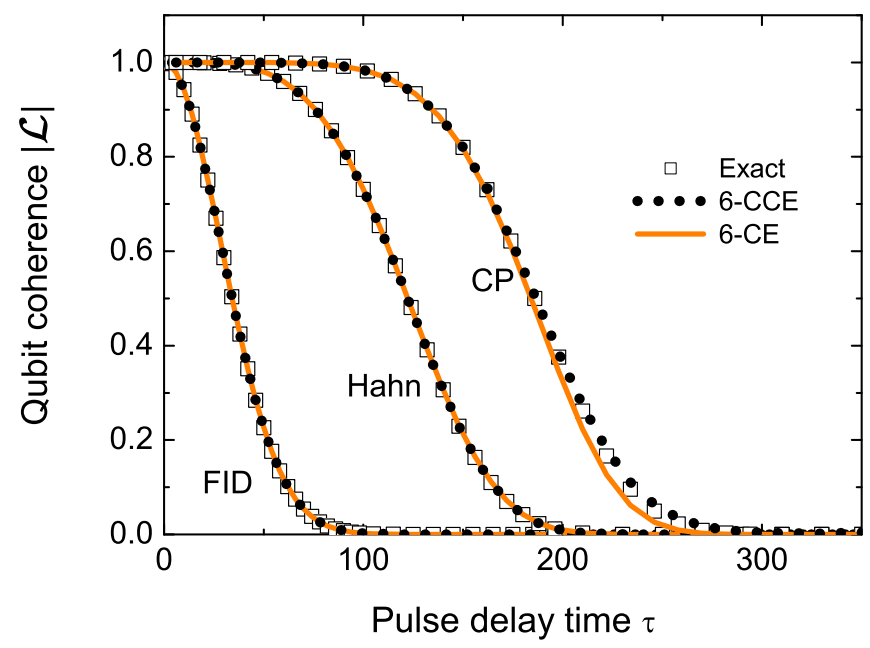

FIG. 5: (Color online). Qubit coherence (empty squares) in freeinduction decay (FID), Hahn echo, and Carr-Purcell echo (CP) for a long "sinusoidal" spin chain with $N=1000$ spins. The results from the 6-CCE (dotted lines) and 6-CE (solid lines) are compared with the exact solutions (empty squares).

pairwise flip-flop between neighboring spins varies smoothly from one end to the other of the chain, so that a correlated cluster can grow to larger and larger size as time passes by. For a time greater than the bath spin flip-flop time $\tau_{\mathrm{sf}}$, the whole bath could become correlated. But the qubit decoherence would be completed within a time $T$ much shorter than $\tau_{\mathrm{sf}}$, if the bath size is relatively large, or

$$
N T^{2} \tau_{\mathrm{sf}}^{-2} \gg 1
$$

due to the large number of small clusters contributing to the decoherence. Under the short-time condition, the CCE can be truncated with a rather small cut-off size $M$. The total contributions from clusters of various sizes to the decay of the qubit coherence as functions of the pulse delay time $\tau$ [see Figs. 2 (b)-(d)] are shown in Fig. 4. For the free-induction decay in Fig. 4(a), the cluster contributions decrease rapidly with increasing the cluster size $m$ at a time much shorter than $\tau_{\text {sf }}$, so the 2-CCE (pair correlation approximation) already converges to the exact result. In the single-pulse Hahn echo [Fig. 4(b)], the higher-order correlations are more noticeable than in the free-induction decay, but the pair correlations still dominate for $\tau \ll \tau_{\text {sf }}$. For the two-pulse Carr-Purcell echo [Fig. 4(c)], as the decoherence due to the pair correlations is eliminated in the leading order of the spin-flip interactions, $\frac{19}{,}$ the larger-size cluster correlations become important and a 6-CCE is required to reproduce the exact solution.

In the relatively large bath, due to the large number of contributing clusters, the qubit decoherence completes when each individual cluster contribution is small, i.e., the small-term condition in Eq. (29) is satisfied. Thus the CCE coincides with the CE. This is verified in Fig. 5 where the exact solution for qubit decoherence agrees with both the CCE and the CE truncated at the 6th order.

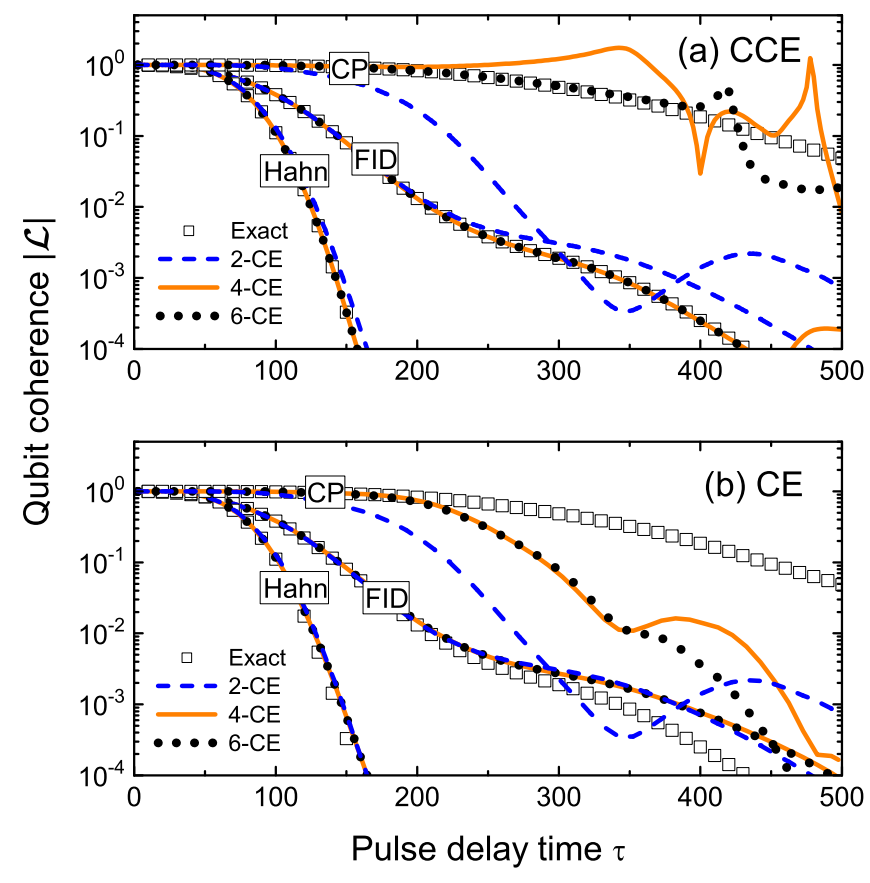

FIG. 6: (Color online) Qubit coherence for a short "sinusoidal" spin chain of $N=100$ spins in free-induction decay (FID), Hahn echo, and Carr-Purcell echo (CP). The results from (a) CCE and (b) CE truncated to the 2nd (dashed lines), the 4th (solid lines) and the 6th (dotted lines) order are compared to the exact solution (empty squares).

\section{B. Small spin bath}

As the second example, we consider a short "sinusoidal" spin chain consisting of $N=100$ spins. In this case the number of contributing clusters is small and the decoherence proceeds much slower. Fig. 6 shows that both the CCE and the $\mathrm{CE}$ converge to the exact result for a time much shorter than $\tau_{\text {sf }}$. As the time approaches and goes beyond the bath spin flip-flop time $\tau_{\mathrm{sf}}$, the deviation from the exact solution is noticeable in both the CCE and the CE, indicating the emergence of correlations for clusters larger than the truncation cut-off size. But the CCE agrees with the exact result as long as it converges, while the CE converges to a different result (for $T=\tau>250$ in free-induction decay, $T=2 \tau>300$ in Hahn echo, and $T=4 \tau>800$ in Carr-Purcell echo). The deviation of the converged CE from the exact result is due to the overlapping correction [see Eq. [30)] neglected in the CE: For $T \gtrsim \tau_{\mathrm{sf}}$, the contribution of an individual cluster could be sizable and the small-term condition in Eq. (29) is violated, so the overlapping correction has to be taken into account.

In the example discussed above, the CCE may not converge at a long time $T \gtrsim \tau_{\text {sf }}$. This is because the qubit-bath spin coupling assumes a smooth sinusoidal distribution and hence the energy cost of each neighboring pair is small and slow-varying as a function of the pair's position along the chain. The small and slow-varying energy cost of a pair-flip makes it possible for one pair-flip to affect its neighboring pairs, then the next neighbors, and so on and so forth. This way large-size cluster 




FIG. 7: (Color online) Qubit coherence in free induction decay for a short "random" spin chain with $N=100$ spins. (a) $M$-CCE and (b) $M$-CE are compared to the exact solution (empty squares).

correlations may grow rapidly after the time goes beyond the pair-flip time $\tau_{\mathrm{sf}}$. In a relatively small bath of smooth qubitbath coupling distribution, the CCE may fail to converge in the long time limit which is of interest in elongating the qubit coherence time by pulse control.

The convergence problem of the CCE for small spin baths may be avoided if the qubit-bath coupling is random so that the bath correlation is localized and the size of correlated clusters is upper bounded. As the last example, we consider a short "random" spin chain consisting of $N=100$ spins. In this case, the pair-flip energy cost is usually much larger than the spin-flip strength unless two neighboring spins are accidentally in near-resonance. Thus large-size cluster correlation can hardly grow significant even for a time well beyond the bath spin flip-flop timescale $\tau_{\mathrm{sf}}$. Figure 7 shows that the pair-correlation approximation already suffices for the qubit decoherence for an arbitrarily long time. Since higher-order correlations are small, the overlapping correction to the CE (which corresponds to at least three-spin cluster correlations) is unimportant, good agreement between the $\mathrm{CE}$ and the exact solution is also seen. Interestingly, in the small bath of random qubit-bath coupling, the qubit decoherence is not fully developed but coherent oscillations persist over a long time. These coherent qubit oscillations have indeed been observed in a system of a similar nature, namely, nitrogen-vacancy centers in diamonds coupled to nuclear spins in the proximity ${ }^{40}$ We would like to point out that the coherent oscillations cannot be reproduced by the LCE truncated as any finite interaction order.

As discussed in Sec. IID the CCE and the CE differ in de-

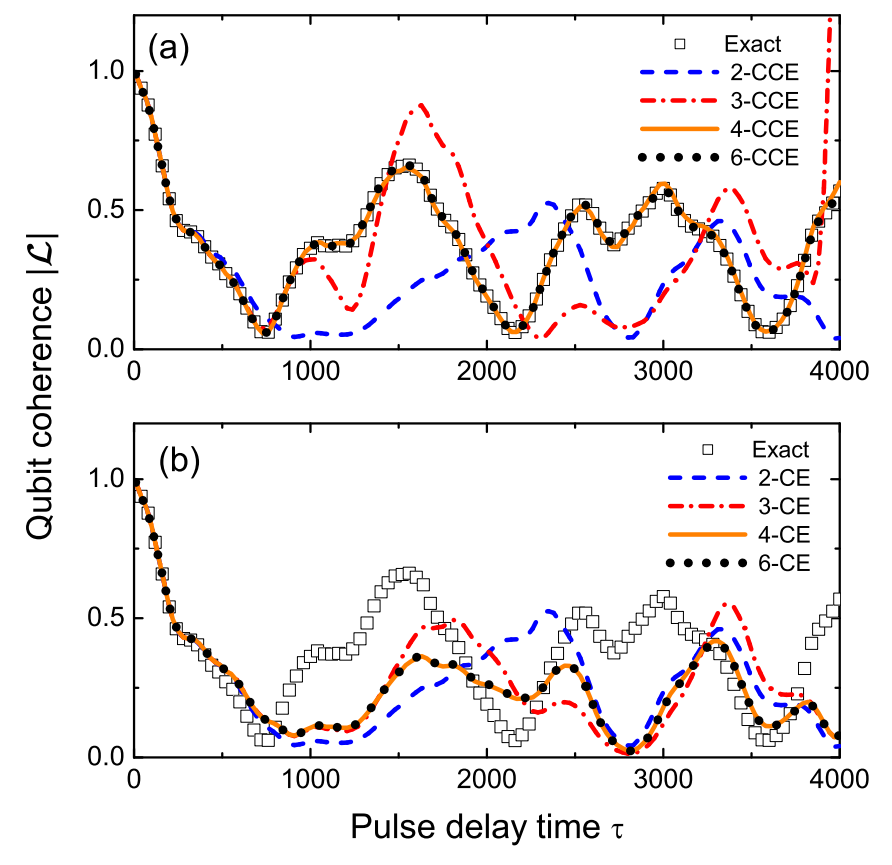

FIG. 8: (Color online) The same as Fig. 7 except that the qubit-bath coupling constants $\left\{z_{n}\right\}$ are such that there are four neighboring spins in near-resonance. Notice that the 4th-order and the 6th-order truncations are not distinguishable by eyes in both CCE and CE.

scribing the multi-spin correlations, which may be important for a small spin bath. Such difference can indeed be seen in Fig. 6 for a short "sinusoidal" spin chain. It is interesting to see the difference between the two theories in studying the coherent multi-spin dynamics in a small bath. For this purpose, we consider a short $(N=100)$ "random" spin chain with accidental near-resonance between four neighboring spins. The pair-flip between the four spins in near-resonance may develop up to four-spin cluster correlations as the evolution time goes beyond the pair-flip timescale $\tau_{\text {sf }}$. Indeed, Fig. 8 8 a) shows coherent oscillations that are correctly reproduced by the CCE at the 4th or higher-order truncation. The correction by the 5th and higher-order clusters is actually negligible, verifying that the dominating cause of the coherent qubit oscillation is due to up to four-spin cluster dynamics in the bath. In contrast, the CE, though already converges at the 4thorder truncation, does not reproduce the exact solution [see Fig. 8 (b)], due to the neglect of the overlapping terms [see Eq. (30)].

\section{CONCLUSION}

We have developed a CCE approach to solving the manybody dynamics of a generic interacting spin bath relevant to the center spin decoherence problem. In this approach, the bath propagator is factorized exactly into the product of cluster correlation terms, each of which accounts for the correlated flip-flops of a group of bath spins. In terms of the standard LCE, a cluster correlation term corresponds to the infinite 
summation of all the connected diagrams with all and only the spins in the cluster flip-flopped. For a finite-time evolution as in qubit decoherence, a convergent result can be obtained by truncating the expansion up to a certain cluster size. The CCE gives exact results whenever it converges. The lowest nontrivial order of the CCE corresponds to the previously developed pair-correlation approximation. Compared to the $\mathrm{CE}$ method, the two theories yield similar results for large spin baths, but for small spin baths only the CCE accurately takes into account the multi-spin cluster correlations. As a simple method to sum over an infinite series of LCE diagrams, the CCE method can be readily applied to the case of interacting bosons or fermions.

\section{Acknowledgments}

This work was supported by Hong Kong RGC Project 2160285 .

\section{APPENDIX A: LINKED CLUSTER EXPANSION FOR SPIN BATH DYNAMICS}

\section{Thermal ensemble LCE}

The LCE for the propagator of an arbitrary spin bath in a thermal ensemble has been derived in Ref. [45]. Here we summarize the main results including the Feynman diagram representation, as the basis of the extension to the LCE for an arbitrary noninteracting bath state.

For a thermal ensemble of spin baths characterized by the noninteracting density matrix $\rho_{0} \equiv e^{-\beta H_{0}} / \operatorname{Tr} e^{-\beta H_{0}}$ with $H_{0}=$ $\sum_{m} \omega_{m} J_{m}^{z}$, the evolution in the contour time-ordered form is

$$
\mathcal{L}^{\mathrm{ens}}=\operatorname{Tr}\left[\rho_{0} \mathscr{T}_{\mathrm{c}} e^{-i \int_{\mathrm{c}} H(t) d t}\right]
$$

Here the contour time-dependent Hamiltonian $H(t)$ switches alternatively between $H^{(+)}$and $H^{(-)}$on the contour [see Fig. 2 (a)]. As an example, the pairwise bath Hamiltonian in Eq. (2) leads to

$$
\begin{aligned}
H(t) & =\frac{\Omega(t)}{2}+\sum_{n} Z_{n}(t) J_{n}^{z}(t)+\sum_{m \neq n} D_{m, n}(t) J_{m}^{z}(t) J_{n}^{z}(t) \\
& +\sum_{m \neq n} B_{m, n}(t) J_{m}^{+}(t) J_{n}^{-}(t),
\end{aligned}
$$

where $\Omega(t), Z_{n}(t), D_{m, n}(t)$, and $B_{m, n}(t)$ depend on which contour time segment $t$ is in, and the spin operators $J_{n}^{z}(t) \equiv J_{n}^{z}$, $J_{n}^{ \pm}(t) \equiv J_{n}^{ \pm}$are time-independent but are written with explicit time-dependence to keep track of the time-ordering.

Eq. A1 can be expanded into series as

$$
\mathcal{L}^{\text {ens }}=\operatorname{Tr}\left[\rho_{0} \sum_{n=0}^{\infty} \frac{(-i)^{n}}{n !} \int_{\mathrm{c}} d t_{1} \cdots \int_{\mathrm{c}} d t_{n} \mathscr{T}_{\mathrm{c}} H\left(t_{1}\right) \cdots H\left(t_{n}\right)\right],
$$

where each term is the sum of ensemble-averaged $\mathscr{T}_{\text {c-products }}$ of spin operators. Consider the ensemble average $\left\langle F_{n}\right\rangle \equiv$
$\operatorname{Tr}\left[\rho_{0} F_{n}\right]$ of an arbitrary product $F_{n} \equiv \mathscr{T}_{\mathrm{c}} J_{m_{1}}^{\alpha_{1}}\left(t_{1}\right) \cdots J_{m_{n}}^{\alpha_{n}}\left(t_{n}\right)$, where the subscripts $m_{q} \in\{1,2, \cdots, N\}$ label bath spins and the superscripts $\alpha_{q} \in\{z,+,-\}$ label the spin operators. In order for $\left\langle F_{n}\right\rangle$ not to vanish, $J^{+}$and $J^{-}$operators must make pairs. An $F_{n}$ consisting of $J^{z}$ operators only is called a fully contracted product, whose ensemble average is trivially evaluated. So next we consider the case with at least one $J^{+}$operator,

$$
F_{n}=\mathscr{T}_{\mathrm{c}} J_{m_{1}}^{\alpha_{1}}\left(t_{1}\right) \cdots J_{m_{k-1}}^{\alpha_{k-1}}\left(t_{k-1}\right) J_{m_{k}}^{+}\left(t_{k}\right) J_{m_{k+1}}^{\alpha_{k+1}} \cdots J_{m_{n}}^{\alpha_{n}}\left(t_{n}\right) .
$$

For the moment, we assume that the product in $F_{n}$ is already time-ordered with $t_{1}>t_{2}>\cdots>t_{n}$ and use the following procedure to reduce $\left\langle F_{n}\right\rangle$ to the sum of ensemble-averaged fully contracted products. First we move the spin raising operator $J_{m_{k}}^{+}\left(t_{k}\right)$ to the right, generating commutators between $J_{m_{k}}^{+}\left(t_{k}\right)$ and the operators on its right,

$$
\begin{aligned}
\left\langle F_{n}\right\rangle & =\operatorname{Tr}\left[\rho_{0} \sum_{p=k+1}^{n} J_{m_{1}}^{\alpha_{1}}\left(t_{1}\right) \cdots\left[J_{m_{k}}^{+}\left(t_{k}\right), J_{m_{p}}^{\alpha_{p}}\left(t_{p}\right)\right] \cdots J_{m_{n}}^{\alpha_{n}}\left(t_{n}\right)\right] \\
& +\operatorname{Tr}\left[\rho_{0} J_{m_{1}}^{\alpha_{1}}\left(t_{1}\right) \cdots J_{m_{k-1}}^{\alpha_{k-1}}\left(t_{k-1}\right) J_{m_{k+1}}^{\alpha_{k+1}}\left(t_{k+1}\right) \cdots J_{m_{n}}^{\alpha_{n}}\left(t_{n}\right) J_{m_{k}}^{+}\left(t_{k}\right)\right] .
\end{aligned}
$$

Then in the second term, we use the cyclic invariance of the trace to move $J_{m_{k}}^{+}\left(t_{k}\right)$ to the left (before $\left.\rho_{0}\right)$, and use $J_{m}^{+}(t) \rho_{0}=e^{\beta \omega_{m}} \rho_{0} J_{m}^{+}(t)$ to move $J_{m_{k}}^{+}\left(t_{k}\right)$ across $\rho_{0}$ back to its original position to get $e^{\beta \omega_{m_{k}}}\left\langle F_{n}\right\rangle$. During this process, we get additional commutators $e^{\beta \omega_{m_{k}} \operatorname{Tr}}\left[\rho_{0} \sum_{p=1}^{k-1} J_{m_{1}}^{\alpha_{1}}\left(t_{1}\right) \cdots\left[J_{m_{k}}^{+}\left(t_{k}\right), J_{m_{p}}^{\alpha_{p}}\left(t_{p}\right)\right] \cdots J_{m_{n}}^{\alpha_{n}}\left(t_{n}\right)\right]$. Collecting all terms, we obtain

$$
\left\langle F_{n}\right\rangle=\operatorname{Tr}\left[\rho_{0} \sum_{p(\neq k)} J_{m_{1}}^{\alpha_{1}}\left(t_{1}\right) \cdots\left[J_{m_{p}}^{\alpha_{p}}\left(t_{p}\right)\right]^{\bullet}\left[J_{m_{k}}^{+}\left(t_{k}\right)\right]^{\bullet} \cdots J_{m_{n}}^{\alpha_{n}}\left(t_{n}\right)\right],
$$

where the contraction between a spin raising operator $J_{m}^{+}(t)$ and an arbitrary spin operator $J_{n}^{\alpha}\left(t_{\alpha}\right)$ is defined as

$$
\begin{aligned}
{\left[J_{n}^{\alpha}\left(t_{\alpha}\right)\right]^{\bullet}\left[J_{m}^{+}(t)\right]^{\bullet} } & \equiv\left[J_{m}^{+}(t)\right]^{\bullet}\left[J_{n}^{\alpha}\left(t_{\alpha}\right)\right]^{\bullet} \\
& \equiv \delta_{m, n} G_{m}\left(t_{\alpha}-t\right)\left[J_{n}^{\alpha}, J_{m}^{+}\right]\left(t_{\alpha}\right),
\end{aligned}
$$

with $G_{m}(t) \equiv \theta(t)\left[1+f\left(\omega_{m}\right)\right]+\theta(-t) f\left(\omega_{m}\right)$ being a Green's function and $f(\omega)=1 /\left(e^{\beta \omega}-1\right)$. Note that the contraction $\left[J_{n}^{\alpha}\left(t_{\alpha}\right)\right]^{\bullet}\left[J_{m}^{+}(t)\right]^{\bullet} \sim\left[J_{n}^{\alpha}, J_{m}^{+}\right]\left(t_{\alpha}\right)$ is still an operator associated with a contour time $t_{\alpha}$ and should be used in subsequent contractions. With $\left[J^{z}, J^{+}\right]=J^{+}$and $\left[J^{-}, J^{+}\right]=-2 J^{z}$, the contraction between $J_{m}^{+}(t)$ and $J_{m}^{z}\left(t_{\alpha}\right)$ [or $J_{m}^{-}\left(t_{\alpha}\right)$ ] eliminates $J_{m}^{+}(t)$ and converts $J_{m}^{z}\left(t_{\alpha}\right)$ [or $\left.J_{m}^{-}\left(t_{\alpha}\right)\right]$ to $J_{m}^{+}\left(t_{\alpha}\right)$ [or $-2 J_{m}^{z}\left(t_{\alpha}\right)$ ], reducing the number of spin operators by one. The resulting product in Eq. A4 is still in a time-ordered sequence, so the timeordering operator can be recovered so that

$$
\begin{aligned}
& \operatorname{Tr}\left[\rho_{0} \mathscr{T}_{\mathrm{c}} J_{m_{1}}^{\alpha_{1}}\left(t_{1}\right) \cdots J_{m_{k-1}}^{\alpha_{k-1}}\left(t_{k-1}\right) J_{m_{k}}^{+}\left(t_{k}\right) J_{m_{k+1}}^{\alpha_{k+1}}\left(t_{k+1}\right) \cdots J_{m_{n}}^{\alpha_{n}}\left(t_{n}\right)\right] \\
& =\operatorname{Tr}\left[\rho_{0} \sum_{p(\neq k)} \mathscr{T}_{\mathrm{c}} J_{m_{1}}^{\alpha_{1}}\left(t_{1}\right) \cdots\left[J_{m_{k}}^{+}\left(t_{k}\right)\right]^{\bullet}\left[J_{m_{p}}^{\alpha_{p}}\left(t_{p}\right)\right]^{\bullet} \cdots J_{m_{n}}^{\alpha_{n}}\left(t_{n}\right)\right] .
\end{aligned}
$$

In the above equation the time ordering $t_{1}>t_{2}>\cdots>t_{n}$ is no longer assumed, since all the spin operators commute in the $\mathscr{T}_{\text {c-product. }}$ 


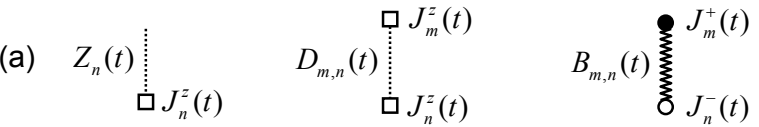

(i) (ii) (iii)

(iv)

(b)

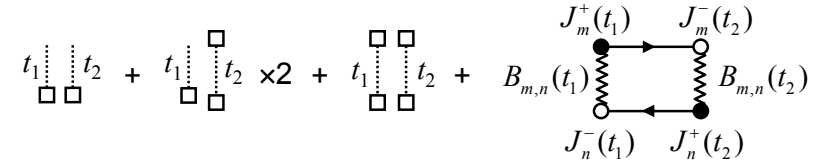

FIG. 9: (a) Diagrammatic representation of the single-spin term $Z_{n}(t) J_{n}^{z}(t)$, the diagonal interaction $D_{m, n}(t) J_{m}^{z}(t) J_{n}^{z}(t)$, and the offdiagonal interaction $B_{m, n}(t) J_{m}^{+}(t) J_{n}^{-}(t)$. (b) Second-order fully contracted diagrams.

The contraction procedure in Eq. A5 can be repeated whenever there is still a spin raising operator left. Thus Wick's theorem follows: Under the average over a noninteracting thermal ensemble, a $\mathscr{T}_{c}$-product of spin operators can be replaced by the sum of all possible fully contracted products which contains only $J^{z}$ operators.

According to Wick's theorem, each term in Eq. (A3) generates a series of fully contracted products. The fully contracted products can be visualized by Feynman diagrams with the following definition of constituent elements and construction rules.

1. A spin operator $J^{+}, J^{-}, J^{z}$ is represented by a vertex as a filled circle, an empty circle, or an empty square, respectively;

2. Each diagonal (off-diagonal) interaction term containing $n$ spin operators in the Hamiltonian is represented by a dashed (wavy) interaction line connecting $n$ vertices;

3. Each contraction $\left[J_{m}^{+}(t)\right]^{\bullet}\left[J_{m}^{\alpha}\left(t_{\alpha}\right)\right]^{\bullet}$ is represented by a solid arrow starting from the vertex $J_{m}^{+}(t)$ and ending at the vertex $J_{m}^{\alpha}\left(t_{\alpha}\right)$. At the end of the propagating arrow the commutator $\left[J_{m}^{\alpha}, J_{m}^{+}\right]$is to be taken;

4. Each $J^{+}(t)$ vertex denoted by a filled circle is connected with one outgoing propagating arrow, each $J^{z}(t)$ vertex denoted by an empty square is either free-standing or connected to one incoming arrow [converting $J^{z}(t)$ to $J^{+}(t)$ ] and one outgoing arrow [from the resulting operator $J^{+}(t)$, and each $J^{-}(t)$ vertex denoted by an empty circle is connected to one incoming arrow [converting $J^{-}(t)$ to $-2 J^{z}(t)$ ] or two incoming arrows [the first arrow converting $J^{-}(t)$ to $-2 J^{z}(t)$, and the second arrow converting $-2 J^{z}(t)$ to $\left.-2 J^{+}(t)\right]$ and one outgoing arrow [from the resulting $J^{+}(t)$.

Note that each fully contracted product (and hence each diagram) is an operator consisting of $J^{z}$ spin operators only.

Taking the Hamiltonian in Eq. (A2) for example, the diagonal single-spin term $Z_{n}(t) J_{n}^{z}(t)$, the diagonal interaction $D_{m, n}(t) J_{m}^{z}(t) J_{n}^{z}(t)$, and the off-diagonal interaction $B_{m, n}(t) J_{m}^{+}(t) J_{n}^{-}(t)$ are visualized in Fig. 9 a). The first-order (a)

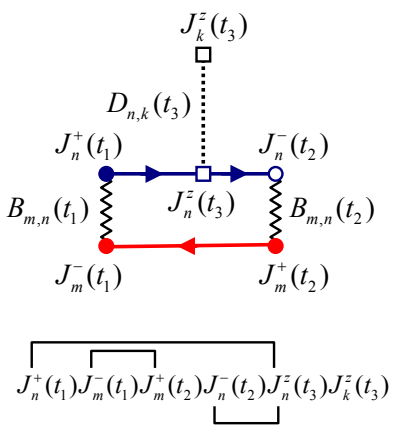

(b)
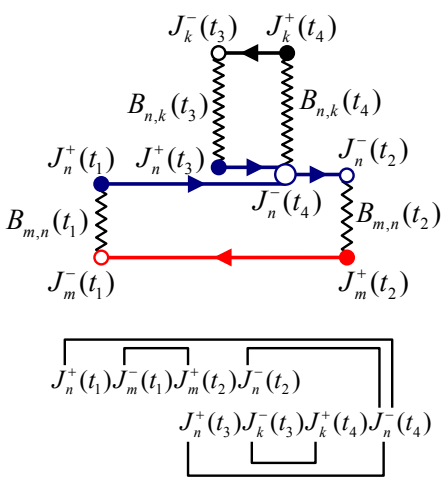

FIG. 10: (Color online) (a) A third-order connected diagram consisting of one diagonal and two off-diagonal interaction terms. (b) A 4th-order connected diagram consisting of four off-diagonal interactions. The contraction processes contained in each diagram are given below.

expansion $(-i) \int_{\mathrm{c}} d t H(t)$ in Eq. (A3) gives two fully contracted products [the first two diagrams in Fig. 9(a)]. The second-order expansion of Eq. (A3) gives four fully contracted products shown in Fig. 9 (b). The first three diagrams

$$
\begin{aligned}
\text { (i) } & =\frac{(-i)^{2}}{2 !} Z_{m}\left(t_{1}\right) Z_{n}\left(t_{2}\right) J_{m}^{z}\left(t_{1}\right) J_{n}^{z}\left(t_{2}\right), \\
\text { (ii) } & =2 \times \frac{(-i)^{2}}{2 !} Z_{m}\left(t_{1}\right) D_{n, l}\left(t_{2}\right) J_{m}^{z}\left(t_{1}\right) J_{n}^{z}\left(t_{2}\right) J_{l}^{z}\left(t_{2}\right), \\
\text { (iii) } & =\frac{(-i)^{2}}{2 !} D_{m, n}\left(t_{1}\right) D_{p, q}\left(t_{2}\right) J_{m}^{z}\left(t_{1}\right) J_{n}^{z}\left(t_{1}\right) J_{p}^{z}\left(t_{2}\right) J_{q}^{z}\left(t_{2}\right),
\end{aligned}
$$

come from diagonal terms and involve no contractions. Here, as a convention, we have suppressed the sum over spin indices and the contour time integrals. The last diagram

$$
\begin{aligned}
\text { (iv) }= & \frac{(-i)^{2}}{2 !} B_{m, n}\left(t_{1}\right) B_{m, n}\left(t_{2}\right) \\
& {\left[J_{m}^{+}\left(t_{1}\right)\right]^{\diamond}\left[J_{n}^{-}\left(t_{1}\right)\right]^{\bullet}\left[J_{n}^{+}\left(t_{2}\right)\right]^{\bullet}\left[J_{m}^{-}\left(t_{2}\right)\right]^{\diamond} }
\end{aligned}
$$

comes from two off-diagonal interaction terms and involves two contractions $\left[J_{m}^{+}\left(t_{1}\right)\right]^{\diamond}\left[J_{m}^{-}\left(t_{2}\right)\right]^{\diamond}$ and $\left[J_{n}^{-}\left(t_{1}\right)\right]^{\bullet}\left[J_{n}^{+}\left(t_{2}\right)\right]^{\bullet}$, as indicated by the two solid arrows in Fig. 9(b) (iv).

Similarly, higher-order diagrams can be constructed by using the above Feynman rules. Figure 10 gives two examples. The 3rd-order diagram in Fig. 10 a) consists of two off-diagonal interactions $B_{m, n}\left(t_{1}\right) J_{n}^{+}\left(t_{1}\right) J_{m}^{-}\left(t_{1}\right)$ and $B_{m, n}\left(t_{2}\right) J_{m}^{+}\left(t_{2}\right) J_{n}^{-}\left(t_{2}\right)$ and one diagonal interaction $D_{n, k}\left(t_{3}\right) J_{n}^{z}\left(t_{3}\right) J_{k}^{z}\left(t_{3}\right)$. It contains three contractions, one on spin $\mathbf{J}_{m}$ and two on spin $\mathbf{J}_{n}$, corresponding to the three solid arrows. The 4th-order one in Fig. 10 b) consists of four off-diagonal interactions and contains five contractions, three on spin $\mathbf{J}_{n}$ and one on each of the other two spins.

To illustrate the evaluation of the diagrams, we consider again the secular pair-interaction Hamiltonian in Eq. (A2) as the example. The rules for constructing the analytical formula for a diagram are:

1. A contour-time dependent constant is associated with each interaction line, namely, $Z_{n}(t)$ for an open-ended 


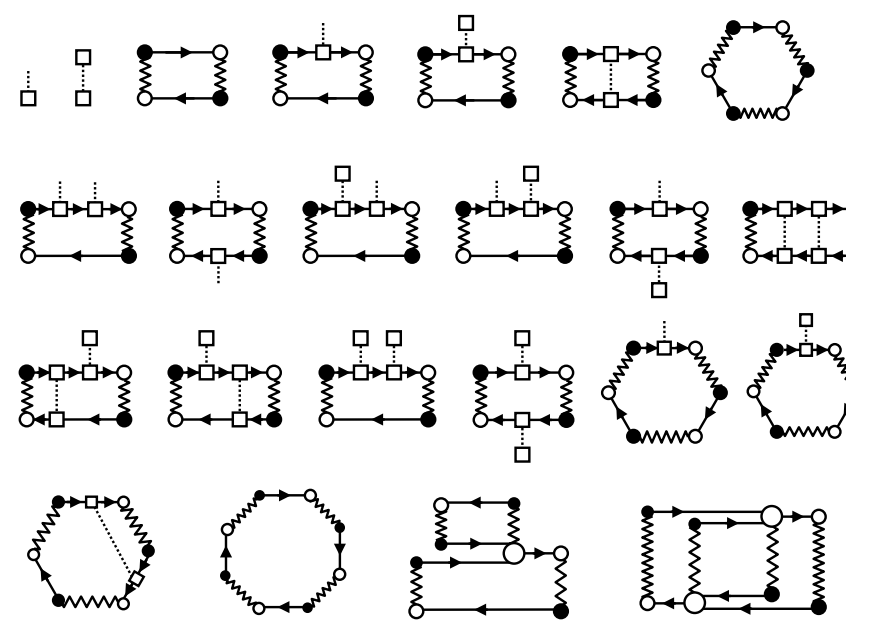

FIG. 11: Topologically inequivalent connected diagrams up to th 4th order for the Hamiltonian in Eq. (2).

dashed line representing the spin splitting, $D_{m, n}(t)$ for a dashed line connecting two diagonal spin operators representing the diagonal interaction, and $B_{m, n}(t)$ for a wavy line representing the off-diagonal interaction;

2. Each solid arrow from $J_{m}^{+}(t)$ to $J_{m}^{\alpha}\left(t_{\alpha}\right)$ gives the Green's function $G_{m}\left(t_{\alpha}-t\right)$, each freestanding $J_{n}^{z}$ vertex gives $J_{n}^{z}$, each $J_{n}^{-}$vertex connected to one incoming arrow gives $(-2) J_{n}^{z}$, and each $J_{n}^{-}$vertex connected with two incoming arrows and one outgoing arrow gives $(-2)$;

3. A global factor $(-i)^{k} / k$ ! is associated with a diagram containing $k$ interaction lines;

4. The spin indices are summed over and the contour times are integrated over.

For example, the last diagram in Fig. 9 b) gives

$\frac{(-i)^{2}}{2 !} B_{m, n}\left(t_{1}\right) B_{m, n}\left(t_{2}\right) G_{m}\left(t_{2}-t_{1}\right) G_{n}\left(t_{1}-t_{2}\right)\left(-2 J_{m}^{z}\right)\left(-2 J_{n}^{z}\right)$,

which can also be evaluated directly from Eq. (A6) by carrying out the two contractions.

Summation of all the fully contracted diagrams leads to the LCE of the ensemble-averaged evolution

$$
\operatorname{Tr}\left(\rho_{0} \mathscr{T}_{\mathrm{c}} e^{-i \int_{\mathrm{c}} H(t) d t}\right)=\operatorname{Tr}\left(\rho_{0} e^{\hat{\pi}}\right),
$$

where $\hat{\pi}$ is the sum of all the connected diagrams, such as the first two diagrams in Fig. 9 (a) and the last diagram in Fig.96), but does not include the disconnected ones such as the first three diagrams in Fig. 9 b). As an example, for the Hamiltonian in Eq. (A2), all the topologically inequivalent connected diagrams up to the 4th order are shown in Fig.11. We see that the number of diagrams increases significantly with increasing perturbation order.

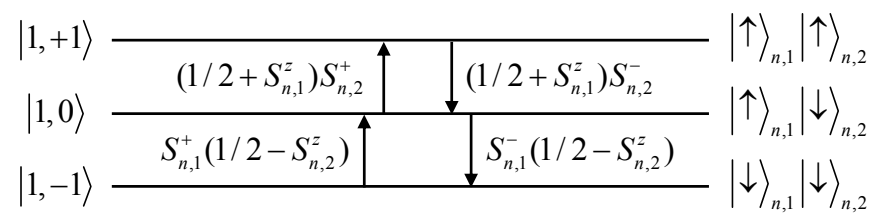

FIG. 12: Mapping of spin-1 operators $J^{ \pm}$to pseudo-spin-1/2 operators. The so-defined mapping restricts the state evolution of the two pseudo-spin-1/2's within the physical Hilbert space of the spin-1.

\section{Single-sample LCE for spin-1/2 baths}

For a single noninteracting bath state $|\mathcal{T}\rangle$ (in contrast to the thermal ensemble), the LCE for spin-1/2 baths has been outlined in Ref. [23]. Here we reproduce the main results using the ensemble LCE depicted in the previous subsection.

The key is that for a spin- $1 / 2$ bath, any noninteracting state $|\mathcal{J}\rangle=\otimes_{n}\left|j_{n}\right\rangle$ can be taken as the ground state of a corresponding noninteracting Hamiltonian,

$$
H_{\mathcal{J}}=\sum_{n} \omega_{n} J_{n}^{z}
$$

where $\omega_{n}<0$ (or $>0$ ) for $\left|j_{n}\right\rangle=|\uparrow\rangle$ (or $|\downarrow\rangle$ ). So the noninteracting single-sample average $\langle\mathcal{J}|O| \mathcal{J}\rangle$ becomes the zerotemperature limit $(\beta \rightarrow+\infty)$ of the corresponding noninteracting ensemble average $\operatorname{Tr}\left(\rho_{\mathcal{J}} O\right)$ with the density matrix $\rho_{\mathcal{J}} \equiv e^{-\beta H_{\mathcal{J}}} / \operatorname{Tr}\left(e^{-\beta H_{\mathcal{J}}}\right)$. In particular, the single-sample expectation value of the bath propagator

$$
\mathcal{L}=\left\langle\mathcal{J}\left|\mathscr{T}_{\mathrm{c}} e^{-i \int_{\mathrm{c}} H(t) d t}\right| \mathcal{J}\right\rangle=\lim _{\beta \rightarrow+\infty} \operatorname{Tr}\left[\rho_{\mathcal{J}} \mathscr{T}_{\mathrm{c}} e^{-i \int_{\mathrm{c}} H(t) d t}\right] .
$$

Thus the single-sample LCE is obtained by simply setting the Green's function $G_{n}(t)=\theta(t) \delta_{j_{n}, \downarrow}-\theta(-t) \delta_{j_{n}, \uparrow}$ and replacing the ensemble average $\operatorname{Tr}\left(\rho_{0} \cdots\right)$ with $\langle\mathcal{J}|\cdots| \mathcal{J}\rangle$, i.e.,

$$
\mathcal{L}=\left\langle\mathcal{T}\left|\mathscr{T}_{\mathrm{c}} e^{-i \int_{\mathrm{c}} H(t) d t}\right| \mathcal{J}\right\rangle=\left\langle\mathcal{J}\left|e^{\hat{n}}\right| \mathcal{J}\right\rangle .
$$

Note that the connected diagrams in $\hat{\pi}$ contain only $J^{z}$ operators, which commute with each other. Thus the single-sample average can be performed for each diagram to convert it into a $c$-number, so that

$$
\mathcal{L}=\exp (\langle\mathcal{J}|\hat{\pi}| \mathcal{J}\rangle) \equiv \exp (\pi) .
$$

\section{Single-sample LCE for higher spins}

For a higher-spin bath, a noninteracting single sample state $|\mathcal{J}\rangle$ in general is not the eigen state of a noninteracting Hamiltonian as in Eq. (A8). Thus the single-sample LCE for higherspin baths may not be derived from the ensemble LCE directly. Here we provide a solution by mapping a higher spin to a composite of pseudo-spin-1/2's. The mapping is such that the physical states form an invariant subspace in the much larger pseudo-spin Hilbert space. 
(a)

a)

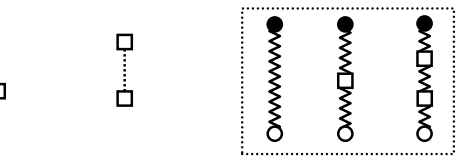

(b)
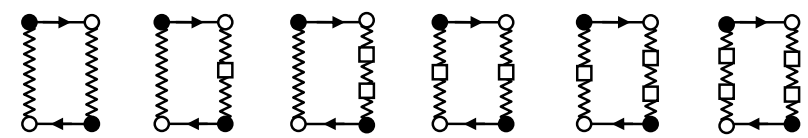

FIG. 13: Diagram representation of (a) $Z_{n} J_{n}^{z}$, (b) $D_{m, n} J_{m}^{z} J_{n}^{z}$, and (c) $B_{m, n} J_{m}^{+} J_{n}^{-}$. (d) Topologically inequivalent second-order connected diagrams for a spin-1 bath.

Without loss of generality, we consider a spin-1 $\mathbf{J}_{n}$. The mapping from the spin-1 states to the states of two pseudospin-1/2's $\mathbf{S}_{n, \lambda}(\lambda=1,2)$ is

$$
\begin{aligned}
|1,-1\rangle_{n} & \rightarrow|\downarrow\rangle_{n, 1}|\downarrow\rangle_{n, 2}, \\
|1,0\rangle_{n} & \rightarrow|\uparrow\rangle_{n, 1}|\downarrow\rangle_{n, 2}, \\
|1,+1\rangle_{n} & \rightarrow|\uparrow\rangle_{n, 1}|\uparrow\rangle_{n, 2},
\end{aligned}
$$

as schematically shown in Fig.12. The two pseudo-spin-1/2's have more basis states than the spin- 1 . To restrict the evolution of the spin state within the physical Hilbert space, we map the spin-1 operators to pseudo-spin-1/2 operators as (see Fig. 12)

$$
\begin{aligned}
J_{n}^{+} & \rightarrow \sqrt{2} S_{n, 1}^{+}\left(1 / 2-S_{n, 2}^{z}\right)+\sqrt{2}\left(1 / 2+S_{n, 1}^{z}\right) S_{n, 2}^{+}, \\
J_{n}^{-} & \rightarrow \sqrt{2} S_{n, 1}^{-}\left(1 / 2-S_{n, 2}^{z}\right)+\sqrt{2}\left(1 / 2+S_{n, 1}^{z}\right) S_{n, 2}^{-}, \\
J_{n}^{z} & \rightarrow S_{n, 1}^{z}+S_{n, 2}^{z},
\end{aligned}
$$

in which the flip-flop of one pseudo-spin- $1 / 2$ is conditioned on the state of the other pseudo-spin- $1 / 2$. Thus each spin- 1 operator is mapped to an interaction term of the two pseudo-spin$1 / 2$ 's. An interaction term between two spin-1's would contain up to four pseudo-spin-1/2 operators, making the Hamiltonian rather complicated. Nonetheless, the LCE can be readily applied to the spin- 1 bath mapped to a pseudo-spin- $1 / 2$ one. For a spin-1 Hamiltonian as in Eq. (A2), the different terms $Z_{n}(t) J_{n}^{z}(t), D_{m, n}(t) J_{m}^{z}(t) J_{n}^{z}(t)$, and $B_{m, n}(t) J_{m}^{+}(t) J_{n}^{-}(t)$ converted to pseudo-spin-1/2 operators, are represented in turn by the three diagrams in Fig. 13 a). There each vertex (denoting a pseudo-spin-1/2 operator according to the same Feynmann rule depicted in Appendix A1] is associated with a physical spin index $n \in\{1,2, \cdots, N\}$ and a pseudo-spin in$\operatorname{dex} \lambda \in\{1,2\}$. The mapping leads to three different types of off-diagonal interactions (including simultaneous interaction involving up to four pseudo-spin-1/2's). As a result, the number of connected diagrams increases dramatically, e.g., we have six topologically inequivalent second-order connected diagrams for spin-1 baths, as shown in Fig. 13 (b), while for a spin-1/2 bath we have only one such diagram (the third diagram in Fig. 11). *rbliu@phy.cuhk.edu.hk

${ }^{1}$ N. V. Prokof'ev and P. C. E. Stamp, Rep. Prog. Phys. 63, 669 (2000).

2 R. Kubo, J. Phys. Soc. Jpn. 9, 935 (1954).

3 D. Pines and C. P. Slichter, Phys. Rev. 100, 1014 (1955).

${ }^{4}$ G. Feher and E. A. Gere, Phys. Rev. 114, 1245 (1959).

5 J. R. Klauder and P. W. Anderson, Phys. Rev. 125, 912 (1962).

${ }^{6}$ D. Loss and D. P. DiVincenzo, Phys. Rev. A 57, 120 (1998).

7 A. Imamoḡlu, D. D. Awschalom, G. Burkard, D. P. DiVincenzo, D. Loss, M. Sherwin, and A. Small, Phys. Rev. Lett. 83, 4204 (1999).

${ }^{8}$ D. Awschalom, D. Loss, and N. Samarth, eds., Semiconductor Spintronics and Quantum Computation (Springer, New York, 2002).

9 I. A. Merkulov, A. L. Elfros, and M. Rosen, Phys. Rev. B 65, 205309 (2002)

10 A. V. Khaetskii, D. Loss, and L. Glazman, Phys. Rev. Lett. 88, 186802 (2002).

11 A. Khaetskii, D. Loss, and L. Glazman, Phys. Rev. B 67, 195329 (2003).

12 W. A. Coish and D. Loss, Phys. Rev. B 70, 195340 (2004).

13 Y. G. Semenov and K. W. Kim, Phys. Rev. B 67, 073301 (2003).

14 R. de Sousa and S. Das Sarma, Phys. Rev. B 67, 033301 (2003).

15 R. de Sousa and S. Das Sarma, Phys. Rev. B 68, 115322 (2003).

16 W. M. Witzel, R. de Sousa, and S. Das Sarma, Phys. Rev. B 72,
161306(R) (2005)

17 W. M. Witzel and S. Das Sarma, Phys. Rev. B 74, 035322 (2006).

18 W. M. Witzel and S. Das Sarma, Phys. Rev. Lett. 98, 077601 (2007).

19 W. M. Witzel and S. Das Sarma, Phys. Rev. B 76, 241303 (2007).

${ }^{20}$ W. Yao, R. B. Liu, and L. J. Sham, Phys. Rev. B 74, 195301 (2006).

21 W. Yao, R. B. Liu, and L. J. Sham, Phys. Rev. Lett. 98, 077602 (2007).

22 R. B. Liu, W. Yao, and L. J. Sham, New J. Phys. 9, 226 (2007).

${ }^{23}$ S. K. Saikin, W. Yao, and L. J. Sham, Phys. Rev. B 75, 125314 (2007).

24 C. Deng and X. Hu, Phys. Rev. B 73, 241303(R) (2006).

25 T. Fujisawa, D. G. Austing, Y. Tokura, Y. Hirayama, and S. Tarucha, Nature 419, 278 (2002).

26 J. M. Elzerman, R. Hanson, L. H. Willems van Beveren, B. Witkamp, L. M. K. Vandersypen, and L. P. Kouwenhoven, Nature 430, 431 (2004).

27 M. Kroutvar, Y. Ducommun, D. Heiss, M. Bichler, D. Schuh, G. Abstreiter, and J. J. Finley, Nature 432, 81 (2004).

28 A. C. Johnson, J. R. Petta, J. M. Taylor, A. Yacoby, M. D. Lukin, C. M. Marcus, M. P. Hanson, and A. C. Gossard, Nature 435, 925 (2005).

29 P.-F. Braun, X. Marie, L. Lombez, B. Urbaszek, T. Amand, P. Renucci, V. K. Kalevich, K. Kavokin, O. Krebs, P. Voisin, et al., 
Phys. Rev. Lett. 94, 116601 (2005).

${ }^{30}$ M. Atatüre, J. Dreiser, A. Högele, K. Karrai, and A. Imamoglu, Science 312, 551 (2006).

${ }^{31}$ F. H. L. Koppens, J. A. Folk, J. M. Elzerman, R. Hanson, L. H. Willems van Beveren, I. T. Vink, H. P. Tranitz, W. Wegscheider, L. P. Kouwenhoven, and L. M. K. Vandersypen, Science 309, 1346 (2005).

32 J. R. Petta, A. C. Johnson, J. M. Taylor, E. A. Laird, A. Yacoby, A. Lukin, C. M. Marcus, M. P. Hanson, and A. C. Gossard, Science 309, 2180 (2005).

33 A. Greilich, D. R. Yakovlev, A. Shabaev, A. L. Efros, I. A. Yugova, R. Oulton, V. Stavarache, D. Reuter, A. Wieck, and M. Bayer, Science 313, 341 (2006).

${ }^{34}$ E. A. Laird, J. R. Petta, A. C. Johnson, C. M. Marcus, A. Yacoby, M. P. Hanson, and A. C. Gossard, Phys. Rev. Lett. 97, 056801 (2006).

35 E. L. Hahn, Phys. Rev. 80, 580 (1950).

${ }^{36}$ G. E. Uhlenbeck and E. Beth, Physica 3, 729 (1936).
37 E. Beth and G. E. Uhlenbeck, Physica 4, 915 (1937).

38 B. Kahn and G. E. Uhlenbeck, Physica 5, 399 (1938).

39 F. Jelezko, T. Gaebel, I. Popa, A. Gruber, and J. Wrachtrup, Phys. Rev. Lett. 92, 076401 (2004).

${ }^{40}$ L. Childress, M. V. G. Dutt, J. M. Taylor, A. S. Zibrov, F. Jelezko, J. Wrachtrup, P. R. Hemmer, and M. D. Lukin, Science 314, 281 (2006).

${ }^{41}$ M. V. G. Dutt, L. Childress, L. Jiang, E. Togan, J. Maze, F. Jelezko, A. S. Zibrov, P. R. Hemmer, and M. D. Lukin, Science 316, 1312 (2007).

42 R. Hanson, V. V. Dobrovitski, A. E. Feiguin, O. Gywat, and D. D. Awschalom, Science 320, 352 (2008).

${ }^{43}$ E. Lieb, T. Schultz, and D. Mattis, Ann. Phys. 16, 407 (1961).

44 Z. Huang, G. Sadiek, and S. Kais, J. Chem. Phys. 124, 144513 (2006).

45 V. G. Vaks, A. I. Larkin, and S. A. Pikin, Sov. Phys. JETP 26, 188 (1968). 